\title{
Evaluación de la eficacia del método de insensibilización por electronarcosis en porcinos ${ }^{\#}$
}

\author{
Assessment of the effectiveness of electrical stunning method in swine
}

\author{
LM González ${ }^{\mathrm{a}}$, MH Romero ${ }^{\mathrm{b} *}$, JA Sánchez ${ }^{\mathrm{b}}$ \\ a Joven investigadora de COLCIENCIAS, Grupo de Investigación en Ciencias Veterinarias CIENVET, \\ Facultad de Ciencias Agropecuarias, Universidad de Caldas, Manizales, Colombia. \\ bDocentes, Grupo de Investigación en Ciencias Veterinarias CIENVET, Departamento de Salud Animal, Programa de Medicina \\ Veterinaria y Zootecnia, Facultad de Ciencias Agropecuarias, Universidad de Caldas, Manizales, Colombia.
}

\begin{abstract}
SUMMARY
National and international sanitary legislation state the guidelines that regulate the slaughter of animals for human consumption purposes due to its implications in animal welfare and meat quality. The aim of this study was to evaluate the slaughter in the swine population of a commercial slaughter plant using behavioural and management indicators. A total of 1,341 pigs were monitored. The electrical stunning method was assessed using behavioural indicators in the stunning box (jumps, falls, vocalizations, slips); the signs of consciousness (blink reflex, righting reflex, vocalizations, pain response) and organizational variables (stunning to bleed interval, collapse of the animal, position of the electrodes, application time of the tongs and effectiveness of the stunning procedure). Vocalizations (26.8\%) were the behavioural response most commonly found and they were associated to the presence of falls and slips $(\mathrm{P}<0.01)$, making evident certain problems related to the handling of the animals and the infrastructure design. Effective stunning was accomplished in $20.6 \%$ of the cases. The righting reflex was positively associated to the presence of all the other signs of consciousness ( $\mathrm{P}$ $<0.01$ ). The duration of the application of the tongs, the stunning to bleed interval and the electrical specifications were not adequate, evidencing several failures in the standardization of the process. It is strongly recommended to incorporate the behavioral and management indicators to audit routinely the efficiency and animal welfare standards during pig slaughter, as well as promoting training of the staff to guarantee a humane procedure.
\end{abstract}

Key words: handling, stunning process, swine behavioural.

\section{RESUMEN}

La legislación sanitaria nacional e internacional contempla lineamientos que regulan el sacrificio de los animales de abasto, por sus implicaciones en el bienestar animal y la calidad de la carne. El objetivo de la investigación fue evaluar la insensibilización y el desangrado de porcinos, por medio de indicadores conductuales y administrativos en una planta de sacrificio del occidente colombiano. Se hizo un seguimiento a 1.341 cerdos insensibilizados por electronarcosis, usando indicadores conductuales en el cajón de insensibilización (caídas, saltos, vocalizaciones, resbalones); signos de sensibilidad (reflejo palpebral, intentos de incorporarse, vocalizaciones, respuesta al dolor) y variables de manejo (tiempo entre insensibilización y el desangrado, colapso del animal, colocación de los electrodos, tiempo de aplicación del método y efectividad de la insensibilización). Las vocalizaciones (26,8\%), fueron las respuestas conductuales más frecuentes y se encontraron asociadas con las caídas y los resbalones $(\mathrm{P}<0,01)$, evidenciando problemas de diseño de la infraestructura y de manejo de los porcinos. La insensibilización efectiva fue alcanzada en un 20,6\% de los cerdos. Los intentos de incorporarse se asociaron con la presencia de todos los signos de sensibilidad $(\mathrm{P}<0,01)$. El tiempo de aplicación del método, el intervalo entre insensibilización y el desangrado, así como la configuración del equipo no fueron adecuadas, evidenciando fallas en la estandarización del proceso. Se sugiere incorporar los indicadores conductuales y de manejo, de forma rutinaria para auditar la eficiencia y los estándares de bienestar animal en plantas de sacrificio de porcinos, así como fortalecer los programas de capacitación del personal para garantizar el sacrificio humanitario.

Palabras clave: comportamiento porcino, manejo, proceso de insensibilización.

\section{INTRODUCCIÓN}

El sacrificio de los animales de abasto público se ha convertido en un tema de importancia científica, social

Aceptado: 06.06.2013

\# Proyecto de investigación financiado por la Vicerrectoría de Investigaciones y Postgrados de la Universidad de Caldas, Manizales, Colombia.

* Calle 65 No. 26-10, Manizales, Caldas, Colombia; marlyn. romero@ucaldas.edu.co y ética en la última década, por su impacto en el bienestar animal (BA) y la calidad de la carne (Smulders y col 2006, Romero y Sánchez 2012). En el primer caso, su relevancia está ligada al papel que se le ha reconocido al BA, como componente del concepto integral de la calidad e inocuidad de la carne (MPS 2007). Desde el punto de vista social y ético, los consumidores demandan altos estándares de BA en la producción de alimentos de origen animal, en especial durante el sacrificio, aspectos que en conjunto orientan las políticas empresariales y legislativas relacionadas con las nuevas tendencias de 
consumo (Horgan y Gavinelli 2006). Los defectos de la calidad de la carne que son influenciados por el sacrificio (insensibilización y sangría) incluyen fracturas de hueso (pelvis y vértebras torácicas), luxación de articulaciones que causan hemorragias (cadera y fémur que dañan el jamón), hemorragias equimóticas por ruptura de capilares, contusiones, sangrado inadecuado y presencia de carne pálida, suave y exudativa (PSE, por sus siglas en inglés) (Traore y col 2012).

En Colombia, la narcosis con gas y la electroinsensibilización o electronarcosis son los dos métodos autorizados para el aturdimiento de los cerdos (MPS 2007). La electronarcosis consiste en el paso de una corriente eléctrica a través de la cabeza del animal, de una magnitud capaz de inducir actividad epiléptiforme tónico/clónica, durante la cual se alcanza el estado de insensibilidad (McKinstry y Anil 2004). Sin importar el método de insensibilización utilizado, este debe causar la inconsciencia instantánea del animal y garantizar este estado hasta la pérdida completa de las reacciones cerebrales como consecuencia del desangrado, de tal modo que no exista sufrimiento por parte del animal durante este proceso (OIE 2012).

La evaluación conductual del animal se ha convertido en una alternativa valiosa, utilizada como un método directo para valorar el BA durante el sacrificio de porcinos (Smulders y col 2006). Su importancia radica en que es un método práctico, poco invasivo y aplicable tanto en condiciones comerciales como científicas, que permiten calificar la eficacia del sacrificio (Grandin 2012). La Unión Europea con el programa "European Union Welfare Quality" y el Servicio de inspección de los Estados Unidos (USDA- Food Safety and Inspection Service), exigen la medición de los indicadores conductuales como requisito de auditoria a nivel empresarial, para la calificación y corrección de los procesos, lo cual se ha traducido en grandes mejoras en el manejo e insensibilización de los animales (Grandin 2012). En lo referente al BA, ponen en evidencia problemas relacionados con inadecuadas prácticas de manejo, diseño de la infraestructura y diferencias en aspectos organizacionales en las plantas de sacrificio (Bourguet y col 2011). El objetivo de este estudio fue identificar problemas de BA durante el proceso de sacrificio en una planta colombiana, utilizando indicadores conductuales y de manejo, así como, determinar si existía relación entre tales indicadores y compararlos con estándares internacionales.

\section{MATERIAL Y MÉTODOS}

\section{MANEJO DE LOS CERDOS}

Se evaluaron 1.341 cerdos en una planta de beneficio comercial autorizada por el Instituto de Vigilancia de Alimentos y Medicamentos (INVIMA) ubicada en el eje cafetero, con un volumen de sacrificio diario promedio de 200 animales (40 cerdos/hora). Los animales se seleccionaron en diferentes días del proceso, durante los meses de marzo, abril y mayo de 2012, de acuerdo con la metodología descrita por Grandin (2012). Los cerdos fueron insensibilizados por electronarcosis en un cajón individual, con dimensiones de $65 \mathrm{~cm}$ x $35 \mathrm{~cm}$ x $18 \mathrm{~cm}$, piso de acero inoxidable y sin sistema de sujeción de cabeza. El equipo se encontraba configurado a $250 \mathrm{~V}, 0,7$ 0,8 A y 60 ciclos de frecuencia, aplicados en la cabeza. En la planta no se realizaba el mantenimiento diario del dispositivo antes de iniciar las operaciones y no se utilizó tábano eléctrico para movilizar los cerdos. La sangría se efectuó en una banda horizontal.

\section{ESTIMACIÓN DE LOS INDICADORES CONDUCTUALES Y DE MANEJO}

La evaluación individual de los indicadores conductuales y de manejo se realizó en el lapso de tiempo comprendido desde el ingreso de los cerdos al cajón de insensibilización, hasta el momento en que inició el aturdimiento, mediante la observación de la presencia o ausencia de los eventos conductuales y de manejo (cuadro 1) (Grandin 2012). Las mediciones fueron hechas por un solo evaluador, para evitar sesgos de información.

\section{EVALUACIÓN DE LA EFECTIVIDAD DE LA INSENSIBILIZACIÓN}

La efectividad de la insensibilización se evaluó teniendo en cuenta la presencia de signos indicativos de la recuperación de la consciencia de los animales, en el intervalo de tiempo desde la salida de los cerdos del cajón de insensibilización hasta el inicio de la sangría. Los signos observados fueron respuesta al dolor, por medio de la aplicación de una aguja en el septum nasal, reflejo palpebral, intentos de incorporarse y las vocalizaciones. La insensibilización se consideró efectiva cuando se presentó la ausencia de estos signos.

\section{ANÁLISIS ESTADÍSTICO}

El análisis se efectúo utilizando el programa Stata Versión 12.0 (College Station, Texas, EU, Licencia Serial 30120546473). Se realizó un análisis descriptivo de las variables (máximos, mínimos, media y DE). Se estudiaron las asociaciones entre las variables continúas mediante regresiones lineales y de las variables categóricas por medio de la prueba $\mathrm{Chi}^{2}$, con un nivel de significancia de $\mathrm{P}<0,05$.

\section{RESULTADOS Y DISCUSIÓN}

Las evaluaciones conductuales integradas con los indicadores de manejo, corresponden a medidas estandarizadas que cuantifican la efectividad y eficiencia del 
Cuadro 1. Indicadores conductuales y administrativos evaluados durante el sacrificio de porcinos.

Behavioral and management indicators assessed during swine slaughter.

\begin{tabular}{|c|c|c|}
\hline Indicador & Definición & Criterios aceptables \\
\hline \multicolumn{3}{|l|}{ Conductuales } \\
\hline Caídas & $\begin{array}{l}\text { Alguna parte del animal, diferente a las pezuñas, } \\
\text { toca el suelo }\end{array}$ & $\begin{array}{c}\text { Excelente } 0 \% \\
\text { Aceptable }<1 \% \\
\text { No aceptable } 1-5 \% \\
\text { Problema grave }>5 \%\end{array}$ \\
\hline Resbalarse & $\begin{array}{l}\text { El animal pierde el equilibrio temporalmente, } \\
\text { interfiriendo con su marcha natural }\end{array}$ & $\begin{array}{c}\text { Excelente } 0 \% \\
\text { Aceptable }<3 \% \\
\text { No aceptable } 3-15 \% \\
\text { Problema grave }>15 \%\end{array}$ \\
\hline Vocalizaciones & Intencionales (excluye jadeos, suspiros o gemidos) & $\begin{array}{c}\text { Excelente } \leq 2 \% \\
\text { Aceptable }<5 \% \\
\text { No aceptable } 5-10 \% \\
\text { Problema grave }>10 \%\end{array}$ \\
\hline De Manejo & & \\
\hline $\begin{array}{l}\text { Tiempo entre insensibilización } \\
\text { y desangrado }\end{array}$ & $\begin{array}{l}\text { Intervalo entre la aplicación del método de } \\
\text { insensibilización y la venopunción }\end{array}$ & $15 \mathrm{~s}$ \\
\hline Colapso del animal & Pérdida de la posición en pie de forma súbita & $100 \%$ \\
\hline Colocación de los electrodos & Electrodos detrás de las orejas & $\begin{array}{c}\text { Excelente } 100 \% \\
\text { Aceptable } 99 \% \\
\text { No aceptable }<99 \% \\
\text { Problema grave }<96 \%\end{array}$ \\
\hline Tiempo de aplicación del método & $\begin{array}{l}\text { Lapso de tiempo en que se aplica la pinza } \\
\text { en la cabeza del cerdo }\end{array}$ & $2-3 \mathrm{~s}$ \\
\hline $\begin{array}{l}\text { Efectividad de la } \\
\text { insensibilización }\end{array}$ & $\begin{array}{c}\text { Presencia de uno o más signos de sensibilidad } \\
\text { durante el desangrado }\end{array}$ & $\begin{array}{c}\text { Excelente } 1 / 2.000 \text { animales } \\
\text { Aceptable } 1 / 1.000\end{array}$ \\
\hline
\end{tabular}

sacrifico, siendo a su vez un indicador del BA durante el proceso (Grandin 2012). Los indicadores de la efectividad del proceso de insensibilización se encontraron por debajo de los estándares recomendados internacionalmente (cuadro 1). La colocación de la pinza de insensibilización fue correcta en el 97,6\% de los animales ( $\mathrm{n}=1.308)$. El tiempo de aplicación del método (promedio 3,07 s $\pm 0,73$ ) fue inferior al recomendado sólo en el 16,5\% de los casos y el intervalo entre la insensibilización y el desangrado fue óptimo $(<15 \mathrm{~s})$ en la mayor proporción de los cerdos observados $(94,8 \%)$. Sin embargo, la ausencia de signos de sensibilidad indicativos de un aturdimiento efectivo se logró sólo en el 20,6\% de los cerdos evaluados, lo cual se considera un problema grave de BA porque la planta no garantizó un sacrificio humanitario. Los resultados obtenidos sugieren que la inefectividad del sacrificio pudo estar relacionada principalmente con fallas en la configuración eléctrica (amperaje, voltaje y la frecuencia de la pinza de insensibilización), el mantenimiento preventivo del equipo antes del inicio de las operaciones y la falta de estandarización del proceso (EFSA 2004). En la planta de estudio el amperaje calibrado en el equipo fue inferior al recomendado para cerdos con pesos comerciales (mínimo 1,25 A, 50-60 ciclos de frecuencia y $250 \mathrm{~V}$ ); el amperaje correcto es responsable de la inducción de la convulsión de tipo gran mal, que asegura la insensibilización inmediata del cerdo (Briese 2000, Grandin 2012). La falla del amperaje del equipo se confirmó cuando se procedió a evaluar los signos de retorno a la sensibilidad, en donde prevaleció el reflejo palpebral positivo $(73,9 \%)$, que es considerado un indiador indirecto de la adecuada calibración del amperaje (A) (Grandin 2012). Para asegurar el correcto funcionamiento de la pinza de aturdimiento y la uniformidad en el tiempo de aplicación de la corriente eléctrica, la planta debería contar con amperímetros y temporizadores. El inadecuado contacto de los electrodos con la piel del cerdo, el grado de hidratación de los porcinos y la cantidad de agua aplicada sobre la piel durante el duchado, son características que pudieron incidir en la falta de conductividad eléctrica del equipo (Grandin 2012). 
Las vocalizaciones $(26,8 \%)$ en el cajón de insensibilización se observaron como una respuesta indicadora de miedo, dolor, o estrés de los cerdos ante el desafío de un ambiente nuevo, porque su presencia se relacionó con los resbalones y las caídas $(\mathrm{P}<0,01)$ (cuadro 2 ), debido a las características del piso (resbaloso y pendiente), a los manejos bruscos por parte del personal y a los problemas de diseño del cajón de insensibilización, el cual carecía de un sistema de sujeción de cabeza (Mota-Rojas y col 2012).

Otro factor relevante para analizar es la interpretación de la observación de los signos de consciencia como indicadores de la efectividad de la insensibilización. Con relación al intento de incorporarse se observó en el 11,9\% de los cerdos; éste corresponde al "reflejo de enderezamiento" y hace parte de las reacciones posturales indicadoras de alteraciones nerviosas en la transmisión de señales al mesencéfalo (Grandin 2012), siendo considerado como un signo confiable y último de retorno completo de la consciencia (EFSA 2004), por lo cual su presencia no puede ser aceptada bajo ninguna circunstancia en la banda de desangrado sin una acción correctiva, aspecto que no se observó en la planta. Su manifestación estuvo significativamente relacionada con la presencia del reflejo palpebral, las vocalizaciones $(4,3 \%)$ y la respuesta al dolor $(9,9 \%)(\mathrm{P}<0,01)$, signos que aparecen gradualmente a medida que aumenta el tiempo entre la insensibilización y el desangrado, o de forma inmediata en un animal donde el método de insensibilización no fue aplicado de forma correcta (Grandin 2012). Aunque la respuesta al dolor y las vocalizaciones probablemente no indican el estado de sensibilidad del animal, una reacción positiva puede relacionarse con la posibilidad eventual del retorno a la consciencia (Briese 2000); en particular la respuesta al dolor evaluada por la aplicación de un pinchazo en el septum nasal, si es negativa indica un plano anestésico grado III, aceptable para la realización de procedimientos dolorosos y estresantes, como el desangrado (Lima-Rodríguez y col 2008).

Los resultados obtenidos permiten concluir que se observaron problemas graves que afectan el BA durante el sacrificio, los cuales se hicieron evidentes mediante el uso de indicadores conductuales y de manejo fáciles de medir e interpretar. Los problemas detectados podrían resolverse mediante la implementación de adecuaciones del cajón de insensibilización, el mantenimiento preventivo y la calibración del equipo de aturdimiento, la capacitación del personal, la sensibilización de la gerencia para implementar programas de BA en la planta de sacrificio y la instauración de un sistema de auditoria que incluya estos indicadores y permitan establecer las mejoras a través del tiempo, entre otros aspectos.

La evaluación de la efectividad de la insensibilización y el desangrado debería ser instaurada en las plantas de beneficio colombianas, por sus implicaciones éticas, comerciales y de seguridad ocupacional; siendo evidente además la necesidad de vincular a la academia en la búsqueda de métodos alternativos de insensibilización irreversible, que contribuyan a garantizar un sacrificio humanitario.

\section{AGRADECIMIENTOS}

Los autores expresan sus agradecimientos a la Vicerrectoría de Investigaciones y Posgrados de la Universidad de Caldas, por la financiación de esta investigación.

Cuadro 2. Frecuencia (\%) de indicadores conductuales evaluados en el cajón de insensibilización.

Frequency $(\%)$ of behavioral indicators assessed in the stunning box.

\begin{tabular}{|c|c|c|c|}
\hline Indicador & Media \pm DE presencia $\%$ & $\begin{array}{c}\text { Escala } \\
\text { (\# eventos) }\end{array}$ & $\begin{array}{l}\text { Observaciones } \\
\mathrm{n}=1.341(\%)\end{array}$ \\
\hline Vocalizaciones & $0,39 \pm 0,77$ & 0 & $982(73,2)$ \\
\hline \multirow[t]{3}{*}{$\mathrm{Si}$} & 26,8 & 1 & $243(18,1)$ \\
\hline & & 2 & $87(6,5)$ \\
\hline & & $\geq 3$ & $29(2,2)$ \\
\hline Resbalones & $0,17 \pm 0,54$ & 0 & $1.179(87,9)$ \\
\hline \multirow[t]{3}{*}{$\mathrm{Si}$} & 12,1 & 1 & $115(8,6)$ \\
\hline & & 2 & $35(2,6)$ \\
\hline & & $\geq 3$ & $12(0,9)$ \\
\hline Caídas & $0,09 \pm 0,32$ & 0 & $1.219(90,9)$ \\
\hline \multirow[t]{2}{*}{$\mathrm{Si}$} & 9,1 & 1 & $116(8,6)$ \\
\hline & & $\geq 2$ & $6(0,5)$ \\
\hline Saltos & $0,07 \pm 0,29$ & 0 & $1.256(93,7)$ \\
\hline \multirow[t]{2}{*}{$\mathrm{Si}$} & 6,3 & 1 & $75(5,6)$ \\
\hline & & $\geq 2$ & $10(0,7)$ \\
\hline
\end{tabular}




\section{REFERENCIAS}

Bourguet C, V Deiss, CC Tannugi, C Terlouw. 2011. Behavioural and physiological reactions of cattle in a commercial abattoir: Relationships with organizational aspects of the abattoir and animal characteristics. Meat Sci 88,158-168.

Briese A. 2000. Evaluation for the return of reflexes (eye reflexes and nose pinch reactions) after electric stunning of slaughter hogs and comparison of electrode positions, variations of time between stunning and bleeding, and different frecuencies of electric current. Doctoral Thesis, Freie Universität Berlin, Berlin, Germany.

EFSA, European Food Safety Authority. 2004. Welfare aspects of the main systems of stunning and killing the main commercial species of animals. EFSA Journal 45, 1-29.

Grandin T. 2012. Recommended Animal Handling Guidelines \& Audit Guide: A systematic Approach to Animal Welfare. AMI Foundation, Washington DC, USA.

Horgan R, A Gavinelli. 2006. The expanding role of animal welfare within EU legislation and beyond. Livest Sci 103, 303-307.

Lima-Rodríguez JR, García-Gil FA, García-García JJ, RochaCamarero G, Martín-Cancho MF, Luis-Fernández L, Crisóstomo V, Usón-Gargallo J, Carrasco-Jiménez MS. 2008. Effects of Premedication with Tiletamine/Xolazepam/Medetomidine during general anesthesia using Se-
voflurane/Fentanyl in swine urdergoing pancreas transplantation. Transplant Proc 40, 3001-3006.

McKinstry JL, MH Anil. 2004. The effect of repeat application of electrical stunning on the welfare of pigs. Meat Sci 67, 121-128.

Mota-Rojas D, M Becerril-Herrera, P Roldan-Santiago, M Alonso-Spilsbury, S Flores-Peinado, R Ramírez-Necoechea, JA Ramírez-Telles, P Mora-Medina, M Pérez, E Molina, E Soní, ME Trujillo-Ortega. 2012. Effects of long distance transportation and $\mathrm{CO}_{2}$ stunning on critical blood values in pigs. Meat Sci 90, 893-898.

MPS, Ministerio de la Protección Social, Colombia. 2007. Resolución 4282 del 21 de noviembre de 2007.

OIE, Organización Mundial de Sanidad Animal. 2012. Código Sanitario para los animales terrestres Título 7. Bienestar de los Animales. 21 ${ }^{\mathrm{a}}$ ed. Paris, Francia.

Romero MH, JA Sánchez. 2012. Bienestar animal durante el transporte y su relación con la calidad de la carne bovina. Rev MVZ Córdoba 17, 2936-2944.

Smulders D, G Verbeke, P Mormède, R Geers. 2006. Validation of a behavioral observation tool to assess pig welfare. Physiol Behav 89, 428-447.

Traore S, L Aubry, P Gatellier, W Przybylski, D Jaworska, K Kajak-Siemaszko,V Santé-Lhoutellier. 2012. Higher drip loss is associated with protein oxidation. Meat Sci 90, 917-924. 
